\title{
A Multicast Approach for UMTS: A Performance Study
}

\author{
Antonios Alexiou, Dimitrios Antonellis, and Christos Bouras
}

Research Academic Computer Technology Institute, N. Kazantzaki str, 26500 Patras, Greece and

Computer Engineering and Informatics Department, University of Patras, 26500 Patras, Greece

alexiua@cti.gr, antonel@ceid.upatras.gr, bouras@cti.gr

\begin{abstract}
In this paper, a multicast scheme for UMTS which only requires insignificant modifications in the current UMTS network infrastructure is analyzed. We analytically present the multicast routing mechanism behind our scheme as well as the multicast group management functionality of it. Furthermore, we present an evaluation of our scheme in terms of its performance. The critical parameters for the evaluation of the scheme are the number of multicast users within the multicast group, the amount of data sent to the multicast users, the density of the multicast users within the cells and the type of transport channel used for the transmission of the multicast data over the air.
\end{abstract}

\section{Introduction}

UMTS constitutes the third generation of cellular wireless networks which aims to provide high-speed data access along with real time voice calls. Wireless data is one of the major boosters of wireless communications and one of the main motivations of the next generation standards [7]. The multicast transmission of real time multimedia data is an important component of many current and future emerging Internet applications, such as videoconference, distance learning and video distribution. It offers efficient multidestination delivery, since data is transmitted in an optimal manner with minimal packet duplication [8].

Compared with multicast routing in the Internet, mobile networks such as UMTS pose a very different set of challenges for multicast. First, multicast receivers are nonstationary and consequently, may change their point of attachment to the network at any given time. Second, mobile networks are generally based on a well-defined tree topology, with the nonstationary multicast receivers being located at the leaves of the network tree. It is therefore not appropriate to apply conventional IP multicast routing mechanisms in UMTS.

Several multicast mechanisms for UMTS have been proposed in the literature. In [1], the authors discuss the use of commonly deployed IP multicast protocols in UMTS networks. However, in [2] the authors do not adopt the use of IP multicast protocols for multicast routing in UMTS and present an alternative solution. The scheme presented in [2] can be implemented within the existing network nodes with only trivial changes to the standard location update and packet-forwarding 
procedures. Furthermore in [3], a multicast mechanism for circuit-switched GSM and UMTS networks is outlined while in [4] an end to end multicast mechanism for software upgrades in UMTS is analyzed. Additionally, the $3^{\text {rd }}$ Generation Partnership Project (3GPP) is currently standardizing the Multimedia Broadcast/Multicast Service (MBMS) [5], [9].

In this paper, we analytically present a multicast scheme for UMTS with the routing mechanism behind the scheme. Additionally, the multicast group management functionality of our mechanism and the performance of the scheme are analyzed. The critical parameters for the evaluation of the scheme are the number of multicast users within the multicast group, the amount of data sent to the multicast users, the density of the multicast users within the cells and the type of transport channel used for the transmission of the multicast data over the air.

\section{A Multicast Approach for UMTS}

In this section we present an overview of a multicast scheme for UMTS. More specifically, the way that the multicast packets are delivered to a group of mobile users is presented in detail. Additionally, we analyze the packet forwarding and routing mechanism behind the multicast scheme as well as the multicast group management functionality of the scheme.

Fig. 1 shows a subset of a UMTS network consisting of eleven multicast users located in six cells. The BM-SC acts as the interface towards external sources of traffic [5]. In the presented analysis, we assume that a data stream coming from an external PDN through BM-SC, must be delivered to these UEs as illustrated in Fig. 1. For the efficient packet forwarding mechanism, every node of the network (except the UEs) maintains a routing list. In these lists of each node, we record the nodes of the next level that the messages for every multicast group should be forwarded.

With multicast, the packets are finally forwarded to those Node Bs that serve multicast users. Therefore, in Fig. 1, the Nodes B2, B3, B5, B7, B8, B9 will receive the multicast packets issued by the BM-SC. We briefly summarize the steps occurred for the delivery of the multicast packets. Before the transmission of the multicast data, the routing lists of the nodes must be filled with useful information. This procedure can be initialized either from the UEs or from the BM-SC (ex. software upgrades). In the former case, consider a UE that decides to become a member of a multicast service. Thus, it sends an appropriate message to the BM-SC requesting this service. Then, every node located in the path between this UE and the BM-SC, when it receives the message from the UE, updates its routing list and forwards the message to the next node. In the second case, the BM-SC initializes this procedure and since it does not have any information regarding the location of the multicast members, a paging procedure at RA and URA level is necessary for the updating of the routing lists.

Consider that the BM-SC receives a multicast packet and forwards it to the GGSN that has registered to receive the multicast traffic. Then, the GGSN receives the multicast packet and by querying its routing list, it determines which downstream SGSCs have multicast users residing in their respective service areas. In Fig. 1, the GGSN duplicates the packet and forwards it to the SGSN1 and the SGSN2. After both destination SGSNs have received the multicast packet and having queried their routing list, 


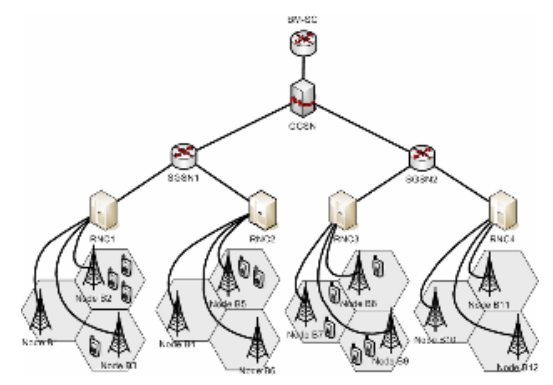

Fig. 1. Packet delivery in UMTS

they determine which RNCs must receive the multicast packet. The destination RNCs receive the multicast packet and send it to the Node Bs that have established the appropriate radio bearers for the multicast application. In Fig. 1, these are Node B2, B3, B5, B7, B8, B9. The transmission of the packets over Uu interface, may be performed on dedicated (DCH) or shared transport channels (ex. High Speed Downlink Shared Channel - HS-DSCH) [7].

\section{Evaluation of the Multicast Scheme}

In this section we present an evaluation, in terms of the telecommunication costs, of the multicast scheme. In particular, we consider a subset of a UMTS network consisting of a single GGSN and $N_{S G S N}$ SGSN nodes connected to the GGSN. Furthermore, each SGSN manages a number of $N_{r a}$ RAs. Each RA consists of a number of $N_{r n c}$ RNC nodes, while each RNC node manages a number of $N_{\text {ura }}$ URAs. Finally, each URA consists of $N_{\text {nodeb }}$ cells. The total number of RNCs and cells are:

$$
\begin{gathered}
N_{R N C}=N_{S G S N} \cdot N_{r a} \cdot N_{r n c} \\
N_{\text {NODEB }}=N_{S G S N} \cdot N_{r a} \cdot N_{r n c} \cdot N_{u r a} \cdot N_{n o d e b}
\end{gathered}
$$

The total transmission cost for packet deliveries is considered as the performance metric. We make a further distinction between processing costs at nodes and transmission costs on links. Similar to [6], there is a cost associated with each link and each node of the network for the packet deliveries. We apply the following notations:

$D_{g s} \quad$ Transmission cost of packet delivery between GGSN and SGSG

$D_{s r} \quad$ Transmission cost of packet delivery between SGSN and RNC

$D_{r b} \quad$ Transmission cost of packet delivery between RNC and Node B

$D_{D C H} \quad$ Transmission cost of packet delivery over the air with DCHs

$D_{H S-D S C H}$ Transmission cost of packet delivery over the air with HS-DSCH

$p_{g} \quad$ Processing cost of packet delivery at GGSN

$p_{s} \quad$ Processing cost of packet delivery at SGSN

$p_{r} \quad$ Processing cost of packet delivery at RNC

$p_{b} \quad$ Processing cost of packet delivery at Node B 
The total number of the multicast UEs in the network is denoted by $N_{U E}$. For the cost analysis, we define the total packets per multicast session as $N_{p}$. Furthermore, network operators will typically deploy an IP backbone network between the GGSN, SGSN and RNC. Therefore, the links between these nodes will consist of more than one hop. Additionally, the distance between the RNC and Node B consists of a single hop $\left(l_{r b}=1\right)$. In the presented analysis we assume that the distance between GGSN and SGSN is $l_{g s}$ hops, while the distance between the SGSN and RNC is $l_{s r}$ hops.

In multicast, the SGSN and the RNC forward a single copy of each multicast packet to those RNCs or Node Bs respectively that serve multicast users. After the correct multicast packet reception at the Node Bs that serve multicast users, the Node Bs transmit the multicast packets to the multicast users via Dedicated or High Speed Shared Transport Channels. The total cost for the multicast scheme is derived from the following equation where $n_{S G S N}, n_{R N C}$ and $n_{N O D E B}$ represent the number of SGSNs, RNCs and Node Bs respectively serving multicast users. The parameter $X$ represents the multicast cost for the transmission of the multicast data over the air.

$$
\begin{aligned}
& M_{S}=\left[p_{g}+n_{S G S N}\left(D_{g s}+p_{s}\right)+n_{R N C}\left(D_{s r}+p_{r}\right)+n_{N O D E B}\left(D_{r b}+p_{b}\right)+X\right] N_{p} \\
& X=\left\{\begin{aligned}
D_{D C H} \cdot N_{U E}, \text { if } & \text { channel }=D C H \\
D_{H S-D S C H} \cdot n_{N O D E B}, \text { if } & \text { channel }=H S-D S C H
\end{aligned}\right.
\end{aligned}
$$

Having analyzed the costs of the multicast scheme, we try to evaluate the cost in function of a number of parameters. The first parameter is the number of the total packets per multicast session $\left(N_{p}\right)$ and the second one is the number of the multicast users $\left(N_{U E}\right)$. We assume a more general network configuration than that illustrated in Fig. 1, with $N_{S G S N}=10, N_{r a}=10, N_{r n c}=5, N_{u r a}=5$ and $N_{\text {nodeb }}=5$.

As we can observe from the equations in the previous section, the cost of the scheme depends on a number of other parameters. Thus, we have to estimate the value of these parameters appropriately, taking into consideration the relations between them. The chosen values of the parameters are presented in Table 1.

Table 1. Chosen parameters' values

\begin{tabular}{cccccccccccc}
\hline$D_{g s}$ & $D_{s r}$ & $D_{r b}$ & $p_{g}$ & $p_{s}$ & $p_{r}$ & $p_{b}$ & $D_{D C H}$ & $D_{H S-D S C H}$ & $l_{g s}$ & $l_{s r}$ & $l_{r b}$ \\
\hline 36 & 18 & 6 & 1 & 1 & 1 & 1 & 5 & 3 & 6 & 3 & 1 \\
\hline
\end{tabular}

In our analysis, the values for the transmission costs of the packet delivery over the air with each of the two transport channels are different. More specifically, the transmission cost over the air with DCHs, is greater than the cost of the packet delivery over the air with HS-DSCH. Therefore, we define the following probabilities for the calculation of the number of the UMTS nodes that serve multicast users:

$P_{S G S N}: \quad$ The probability that an SGSN serve multicast users

$P_{R N C}$ : The probability that an RNC (served by an SGSN with multicast users), serves multicast users

$P_{\text {NODEB }}: \quad \begin{aligned} & \text { The probability that a } \\ & \text { serves multicast users }\end{aligned}$ 
For the cost analysis, we assume that $P_{S G S N}=0.4, P_{R N C}=0.3$ and $P_{N O D E B}=0.4$. Consequently, the number of the SGSNs, the RNCs and the Node Bs that serve multicast users are $n_{S G S N}=N_{S G S N} P_{S G S N}=4, n_{R N C}=N_{R N C} P_{S G S N} P_{R N C}=60$ and $n_{N O D E B}=N_{N O D E B}$ $P_{S G S N} P_{R N C} P_{N O D E B}=600$ respectively.

Fig. 2a presents the cost of the multicast scheme in function of the $N_{p}$ for different transport channels (DCH and HS-DSCH) used for the transmission of the multicast data over the air. The y-axis presents the total cost of the multicast scheme, while the $\mathrm{x}$-axis shows the total packets per multicast session. Regarding the use of DCHs, in Fig. 2a, we have calculated the costs for three different values of the number of multicast users, indicating that the multicast cost increases rapidly when the amount of the multicast data increases. Furthermore, for a given $N_{p}$, the multicast cost increases as the members of the multicast group increase. This occurs because the greater the number of multicast users is, the greater the number of DCHs needed for the transmission of the multicast data over the air. Additionally, eqn (3) shows that in case of HS-DSCH, the cost of the multicast scheme depends only on the number of packets per multicast session. This can be shown in Fig. 2a where we can observe that the greater the $N_{p}$ is, the greater the multicast cost becomes.

Another interesting parameter is the $P_{N O D E B}$. Assuming that $N_{U E}=1500, N_{p}=500$, we can calculate the cost for the multicast scheme, for the transport channels we use. Fig. $2 \mathrm{~b}$ presents the cost of the multicast scheme in function of $P_{N O D E B}$ for different transport channels. It is obvious from Fig. $2 b$ that the cost of the multicast scheme is decreased as $P_{N O D E B}$ converges to zero. This means that the greater the number of

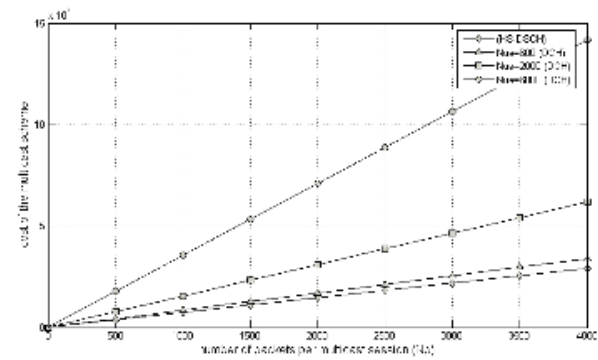

a)

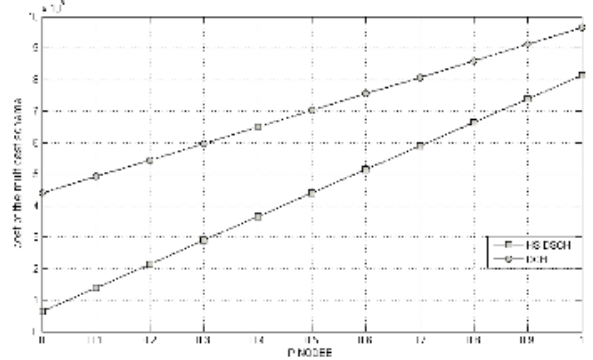

b)

Fig. 2. Costs of the multicast scheme against $N p$ and $P_{N O D E B}$ for different transport channels

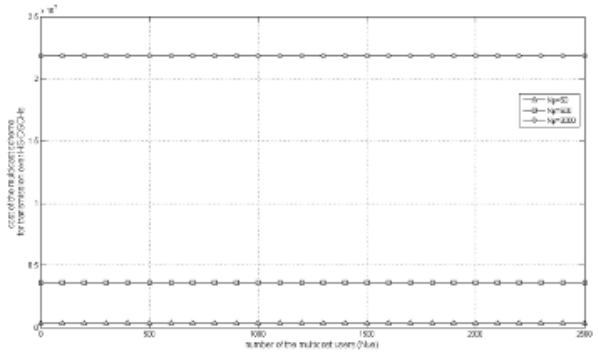

a)

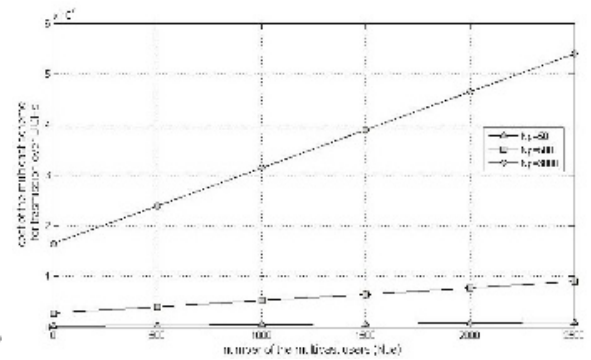

b)

Fig. 3. Costs of the multicast scheme against $N_{U E}$ using different transport channels 
multicast users per cell is, the lower the cost of the multicast scheme is. Furthermore as Fig.2b indicates, the use of HS-DSCHs is absolutely preferable than the DCHs.

Furthermore, we try to estimate the cost of the multicast scheme in function of the $N_{U E}$ (Fig. 3). As we observe, three different values of the number of the total packets per multicast session $\left(N_{p}\right)$ have been calculated. Fig. 3a presents the cost of the multicast scheme against $N_{U E}$ in case we use HS-DSCH for the transmission of the multicast data over the air. According to Fig. 3a, the cost of the multicast scheme is independent from the number of multicast users in case of HS-DSCH. The cost of the multicast scheme in this case depends mainly on the number of Node Bs that serve multicast users. Only one per cell HS-DCSH is established and it is capable of supporting a great number of multicast users in the specific cell. Regarding the multicast cost against $N_{U E}$ in case of using DCHs for the transmission of the multicast data over the air, the relation between them is predictable, since the greater the number of the multicast UEs is, the greater the cost becomes (Fig. 3b).

\section{Conclusions and Future Work}

In this paper, we have presented a multicast scheme for UMTS. We have analyzed the delivery of the multicast packets to a group of mobile users and the performance of such a delivery in terms of the telecommunication cost. Considering a general network configuration, we have presented the cost of a multicast scheme in function of a number of parameters. The step that follows this work is to implement the above presented multicast scheme in NS-2 simulator and confirm the relation of the costs through the experiments.

\section{References}

1. Hauge, M., Kure, O.: Multicast in $3 \mathrm{G}$ networks: Employment of existing IP multicast protocols in UMTS. in Proc. WoWMoM 2002 96-103

2. Rummler, R., Chung, Y., Aghvami, H.: Modeling and Analysis of an Efficient Multicast Mechanism for UMTS. IEEE Trans. on Vehicular Technology, vol. 54, no. 1(2005) 350-365

3. Lin, Y.: A multicast mechanism for mobile networks. IEEE Communication Letters, vol. 5 (2001) 450-452

4. Rummler, R., Aghvami, H.: End-to-end IP multicast for software upgrades of reconfigurable user terminals within IMT-2000/UMTS networks. IEEE ICC'02, vol. 1 (2002) 502-506

5. 3GPP, TS 23.246, Multimedia Broadcast/Multicast Service (MBMS); Architecture and functional description, V6.7.0

6. Ho, J. S., Akyildiz, I. F.: Local anchor scheme for reducing signaling costs in personal communications networks. IEEE/ACM Trans. Networking, vol. 4 (1996) 709-725

7. Holma. H.., Toskala, A.: WCDMA for UMTS: Radio Access for Third Generation Mobile Communications. John Wiley \& Sons (2003)

8. Gossain, H., Cordeiro, C. Argawal, D.: Multicast: Wired to Wireless. IEEE Communications Magazine (2002) 116-123

9. 3GPP, TS 22.146, Technical Specification Group Services and System Aspects; Multimedia Broadcast/Multicast Service, Stage 1 (Release 6) 